\title{
EFEITO DO FOTOPERÍODO NO CRESCIMENTO, CONSUMO ALIMENTAR E QUALIDADE DA CARCAÇA DE TILÁPIAS INVERTIDAS
}

\author{
Rodrigo Diana Navarro ${ }^{1}$ \\ Thalita Milo Simões Ferreira ${ }^{1}$ \\ Fernanda Keley Silva Pereira Navarro ${ }^{2}$
}

\begin{abstract}
NAVARRO, R. D.; FERREIRA, T. M. S.; NAVARRO, F. K. S. P. Efeito do fotoperíodo no crescimento, consumo alimentar e qualidade da carcaça de tilápias invertidas. Arq. Ciênc. Vet. Zool. UNIPAR, Umuarama, v. 19, n. 2, p. 77-80, abr.jun. 2016.

RESUMO: A incidência luminosa pode induzir diversos efeitos sobre o crescimento, alimentação, desenvolvimento e reprodução de peixes. O fotoperíodo é sinal principal para maior influência sobre o ritmo circadiano dos peixes e pode afetar o ganho de peso, a ingestão de alimento e a utilização de energia. O objetivo deste trabalho foi avaliar os efeitos de diferentes regimes de fotoperíodo no crescimento, consumo alimentar e qualidade da carcaça de tilápias invertidas, em um sistema fechado. Foram utilizados 150 alevinos de tilápias invertidas, provenientes da estação de piscicultura. Os peixes foram acondicionados e distribuídos em 15 aquários com a capacidade de 65 litros cada um, em delineamento inteiramente ao acaso, com três tratamentos e cinco repetições. Os tratamentos diferenciarão nas simulações dos fotoperíodos emerais (horas de luz) que durarão $\mathrm{T} 1=24$ horas de escuro, $\mathrm{T} 2=12$ horas de escuro e 12 horas de Luz, $\mathrm{T} 3=24$ horas de luz. Cada peixe foi considerado uma unidade experimental. Não houve diferença significativa para desempenho, mas houve diferença para Índice gonadossomático (IGS). Conclui-se que o fotoperíodo (12L:12E) promove melhor índice gonadossomático em tilápia. Para melhor qualidade de carcaça e desempenho produtivo sugere o fotoperíodo (24L:0E).
\end{abstract}

PALAVRA-CHAVE: Escuro. Fotoperiodo. Oreochomis niloticus. Ritmo biológico.

\section{PHOTOPERIOD EFFECT ON GROWTH, FEED INTAKE AND CARCASS OUALITY REVERSED TILAPIA}

ABSTRACT: The light incidence can induce different effects on growth, feeding and development and reproduction of fish. The photoperiod is the main signal for greater influence on the circadian rhythm of the fish and can affect weight gain, feed intake and energy use. This study aimed to demonstrate the effects of different photoperiodic regimes on growth, feed intake and carcass tilapia quality reversed in a closed system. 150 were used fry reversed tilapia, from fish farming station. The fish were packed and distributed in 15 tanks with a capacity of 65 liters each, in a completely randomized design with three treatments and five repetitions. Treatments differentiate in the simulations of emerais photoperiod (light hours) that will last T1 $=24$ hours dark, T2 = 12 hours of darkness and 12 hours light, T3 = 24 hours of light. Each fish was considered an experimental unit. There was no significant difference in performance, but there were differences for IGS. I conclude that the photoperiod (12L: 12D) promotes better GSI in tilapia. To better carcass quality and growth performance suggests the photoperiod (24L: 0D).

KEYWORDS: Biological rhythm. Dark. Oreochomis niloticus. Photoperiod.

\section{EFECTO DE FOTOPERIODO EN EL CRECIMIENTO, CONSUMO ALIMENTAR Y CALIDAD DE CAPARAZÓN DE TILAPIAS INVERTIDAS}

RESUMEN: La incidencia de luz puede inducir diversos efectos sobre el crecimiento, alimentación, desarrollo y reproducción de peces. El fotoperiodo es la señal principal de una mayor influencia en el ritmo circadiano de los peces y puede afectar la ganancia de peso, la ingestión de alimento y el consumo de energía. El objetivo de esta investigación ha sido demostrar los efectos de diferentes regímenes de fotoperiodo sobre el crecimiento, consumo alimentar y calidad de caparazón de tilapias invertidas, en un sistema cerrado. Se utilizaron 150 alevines de tilapias invertidas, provenientes de la estación de piscicultura. Los peces fueron acondicionados y distribuidos en 15 tanques con una capacidad de 65 litros cada uno, en un diseño completamente al azar, con tres tratamientos y cinco repeticiones. Los tratamientos se diferencian en las simulaciones de fotoperiodos emerales (horas de luz) que durarán T1 $=24$ horas de oscuridad, $\mathrm{T} 2=12$ horas de oscuridad y 12 horas de luz, $\mathrm{T} 3=24$ horas de luz. Cada pez fue considerado una unidad experimental. No hubo diferencia significativa en el rendimiento, pero hubo diferencia para el Índice Gonadosomático (IGS). Se concluye que el fotoperiodo (12L: 12D) promueve mejor GSI en tilapia. Para mejor calidad de caparazón y rendimiento de la producción se sugiere fotoperiodo (24L: 0E).

PALABRAS CLAVE: Fotoperiodo. Oreochomis niloticus. Oscuro. Ritmo biológico.

DOI: https://doi.org/10.25110/arqvet.v19i2.2016.5924

'Laboratório de Aquicultura e de Biotecnologia de Organismos Aquáticos da Universidade de Brasília, Faculdade de Agronomia e Medicina Veterinária, Brasília, DF, Brazil. email: navarrounb@gmail.com

${ }^{2}$ Instituto Federal de Educação Ciência e Tecnologia de Goiás, Aguas Lindas de Goiás, GO, Brazil. 


\section{Introdução}

Os movimentos de rotação e translação da terra fazem com que os organismos que nela habitam sejam submetidos a mudanças cíclicas dos fatores ambientais. Todas as formas de vida respondem aos ciclos do sol, da lua e das estações, sendo denominados de relógio biológico ou ritmo circadiano. A maioria dos eventos bioquímicos, fisiológicos e comportamentais dos seres vivos são rítmicos. Os fatores externos ao relógio de natureza rítmica que influenciam nos ritmos biológicos chamam-se sincronizadores ou "zeitgebers". Os ciclos de fotoperíodo e a temperatura são os principais sincronizadores dos ritmos diários e anuais (AGOSTINHO et al., 1984; ADAMANTE, 2005; NAVARRO et al., 2014).

O efeito de variáveis ambientais sobre o crescimento, sobrevivência e as respostas fisiológicas dos peixes têm sido extremamente estudadas. Alguns estudos científicos e investimentos estão sendo aplicados na área de piscicultura, com a finalidade de minimizar o estresse dos peixes decorrente de diversos fatores como manejo, estado nutricional, qualidade da água, temperatura, fotoperíodo, salinidade (AGOSTINHO et al., 1984; ALMAZAN RUEDA et al., 2005; NAVARRO; NAVARRO, 2012; NAVARRO et al., 2015).

A iluminação corresponde a um dos diversos estímulos ambientais que, em condições de criação, pode ser facilmente manipulada nos sistemas de recirculação de água a fim de alterar aspectos fisiológicos, crescimento, sistema neuro-hormonal, reprodução, comportamento. A diversidade de respostas à luz entre os peixes pode ser reflexo de adaptações específicas ao seu ambiente, onde a luz pode variar em termos de quantidade (intensidade), qualidade (espectro) e duração (fotoperíodo) (BALDISSEROTTO, 2002; AMANO, 2000; 2004, NAVARRO et al., 2013).

Fatores extrínsecos são particularmente importantes no crescimento de vertebrados ectotérmicos, como os peixes teleósteos, que dependem da temperatura, fotoperíodo e disponibilidade de alimentos para os processos iniciais de desenvolvimento (TAYLOR et al., 2005). A manipulação do fotoperíodo com o interesse de melhorar o crescimento dos peixes tem se tornado uma área de interesse dentro da produção comercial de várias espécies (TAYLOR; MIGAUD 2009). O fotoperíodo ótimo para o crescimento dos peixes pode variar em relação à espécie, idade ou fase de desenvolvimento, estação e temperatura ambiente (BANI et al., 2009)

A luz pode induzir efeitos sobre o crescimento de várias espécies de peixes. Em salmonídeos, o fotoperíodo atua diretamente sobre o crescimento, por meio de sua influência sobre ritmos endógenos (ENDAL et al., 2000) e por intermédio de fotoestimulação direta do eixo somatotrófico (FÁLCON et al., 2010). Sabe-se que GH e IGF-1, particularmente, são potentes estimuladores do crescimento muscular. Rápidos aumentos dos níveis circulantes de GH e IGF-1 têm sido relacionados com altas temperaturas e dias longos.Objetivou-se avaliar a influência do fotoperíodo no crescimento, consumo alimentar e na qualidade da carcaça de tilápias invertidas.

\section{Material e Métodos}

O experimento foi realizado no laboratório de aquicultura da Universidade de Brasília UnB com duração de 60 dias. O estudo foi aprovado pelo Comitê de Ética da Universidade de Brasília (protocolo CEUA / UnB 107251/2012).

Foram utilizados 150 alevinos de Tilápia (Oreochomis niloticus), machos invertidos com peso inicial médio de $16,65 \pm 1,65$ gr. Dados de peso, comprimento total, referente a cada peixe, dos diferentes tratamentos foram mensurados por meio de medição com paquímetro e balança de precisão $(0,001 \mathrm{~g})$. Os peixes foram acondicionados e distribuídos em 15 aquários de 65 litros cada um, em um sistema de recirculação em que a água do aquário foi coletada e filtrada através de um filtro mecânico e biológico instalado em caixa d'água de $500 \mathrm{~L}$ para uso posterior, a fim de manter a qualidade da água do sistema. $\mathrm{O}$ delineamento experimental foi inteiramente ao acaso, com três tratamentos e cinco repetições. Os tratamentos diferenciaram nas simulações dos fotoperíodos T1=0L:24E, T2=24L:0E, T3 =12L:12E. Cada aquário foi considerado uma unidade experimental.

O controle das horas de luz fornecidas durante o experimento foi feito por "timers" automáticos que apagaram e acenderam as luzes das bancadas durante os períodos estipulados. Os tratamentos foram isolados para evitar a incidência de luz proveniente de outras fontes luminosas durante o período de escuro de cada tratamento, a fim de padronizar a luz dentro de cada tratamento.

Foram mensurados os parâmetros de qualidade de água: oxigênio dissolvido, $\mathrm{pH}$, temperatura e condutividade elétrica. Os níveis de oxigenação foram mantidos com auxílio de aeradores e mensurados por meio de um oxímetro. $\mathrm{O}$ $\mathrm{pH}$ foi medido através do peagâmetro, a temperatura com o uso de termômetro digital e a condutividade elétrica através do condutivímetro.

O manejo alimentar foi realizado utilizando uma ração comercial, contendo $36 \%$ de $\mathrm{PB}$ e $3.100 \mathrm{kcal}$ de ED/ $\mathrm{kg}$. A alimentação foi dividida em duas refeições diárias com intervalo de 7 horas entre a primeira e a segunda refeição. A ração foi fornecida às 9:00 e às 16:00 horas. Após 15 minutos de cada refeição, as caixas foram sinfonados para a retirada das sobras, e mensuração do consumo de ração.

Para a determinação da composição química dos peixes, todos os peixes foram anestesiados com benzocaína $200 \mathrm{mg} / \mathrm{L}$, eutanasiados e em seguida as carcaças foram inteiramente moídas e homogeneizadas para determinação da umidade, proteína bruta, extrato etéreo, de acordo com a Association of Official Analytical Chemists - AOAC (1990).

Foram analisados os parâmetros nutricionais de ganho de peso (GP), consumo aparente de ração (CR), Peso final (PF), comprimento total e padrão (CT e CP).

As análises estatísticas foram feitas com o auxílio do programa SAS (1997), sendo as médias dos tratamentos comparadas pelo teste de Duncan a 5\% de significância.

\section{Resultados e Discussão}

Os parâmetros de qualidade de água apresentaram os seguintes valores médios: $21,74 \pm 0,43^{\circ} \mathrm{C}$ para temperatura inicial, $29,50 \pm 0,03^{\circ} \mathrm{C}$ para temperatura ambiente, 5,5 para $\mathrm{pH} ; 6,1 \pm 0,36 \mathrm{mg}$.L-1, oxigênio dissolvido, para amô- 
nia valor de 0,35 e 0,10 para $\mathrm{NO}_{2}$ de acordo com Navarro et al. (2012).

Durante os 60 dias de experimento não foram observados diferenças significativas para peso final, comprimento padrão, ganho de peso e ganho de peso aos 30 dias. Já o Ganho de peso aos 60 dias foi observado diferença significativa para tratamento com $24 \mathrm{~L}: 0 \mathrm{E}$, esse mesmo tratamento foi observado um consumo maior entre os tratamentos, provavelmente, aos 60 dias de experimento, os animais utilizaram sua energia da dieta para ganho de peso (Tabela 1 e Gráfico 1). Resultados semelhantes a este em que os pesquisadores verificaram uma relação positiva entre exposição à luz contínua crescimento somático e ingestão alimentar. Esta relação foi observada em salmão do Atlântico (OPPEDAL et al., 1997) em bacalhau do Atlântico, Gadus morhua (HANSEN et al., 2001; TARANGER et al., 2006), em Melanogrammusa eglefinus (DAVIE et al., 2007) e Pagrus major (BISWAS et al., 2010) e Sparus aurata (GINÉS et al., 2004).

Tabela 1: Média \pm erro padrão ou desvio padrão do Peso final $(\mathrm{g})$, Comprimento total (cm), Comprimento padrão (cm), Ganho de peso (GP), Ganho de peso aos 30 dias (GP 30), Ganho de peso aos 60 dias (GP 60) machos de tilápia em função do fotoperíodo.

\begin{tabular}{|c|c|c|c|}
\hline Tratamento & 24L:0E & 12L:12E & 0L:24E \\
\hline Peso final (g) & $48,42 \pm 11,63^{\mathrm{a}}$ & $45,46 \pm 11,21^{\mathrm{a}}$ & $44,80 \pm 17,07^{\mathrm{a}}$ \\
\hline Comprimento total $(\mathrm{cm})$ & $14,08 \pm 1,18^{a}$ & $13,45 \pm 1,30 \mathrm{ab}$ & $12,90 \pm 1,89^{b}$ \\
\hline Comprimento padrão $(\mathrm{cm})$ & $10,35 \pm 1,62^{a}$ & $9,71 \pm 1,54^{\mathrm{a}}$ & $9,60 \pm 0,84^{\mathrm{a}}$ \\
\hline Ganho de peso(g) & $32,41 \pm 11,63^{\mathrm{a}}$ & $30,83 \pm 11,21^{\mathrm{a}}$ & $31,38 \pm 17,07^{\mathrm{a}}$ \\
\hline Ganho de peso 30 dias(g) & $6,89 \pm 0,02^{\mathrm{a}}$ & $6,97 \pm 0,03^{\mathrm{a}}$ & $8,28 \pm 0,03^{\mathrm{a}}$ \\
\hline Ganho de peso aos 60 dias (g) & $37,35 \pm 0,02^{\mathrm{b}}$ & $29,69 \pm 0,03^{a}$ & $32,82 \pm 0,03^{\mathrm{a}}$ \\
\hline
\end{tabular}

*Médias seguidas de letras diferentes diferem a $5 \%$ de probabilidade pelo teste de Duncan.

Tabela 2. Média \pm erro padrão ou desvio padrão do índice vicerossomático (IVS), índice hepatossompárica (IHS), Peso da gônada (PG) e Índice gonadossomático (IGS) de machos de tilápia em função do fotoperíodo

\begin{tabular}{cccc}
\hline Tratamento & 24L:0E & $\mathbf{1 2 L}: 12 E$ & 0L:24E \\
\hline IVS & $9,12 \pm 1,96^{\mathrm{a}}$ & $9,76 \pm 1,98^{\mathrm{a}}$ & $9,75 \pm 2,57^{\mathrm{a}}$ \\
IHS & $2,35 \pm 1,34^{\mathrm{a}}$ & $2,27 \pm 0,76^{\mathrm{a}}$ & $2,27 \pm 0,75^{\mathrm{a}}$ \\
PG & $0,51 \pm 0,40^{\mathrm{a}}$ & $0,66 \pm 0,40^{\mathrm{a}}$ & $0,40 \pm 0,66^{\mathrm{a}}$ \\
IGS & $1,06 \pm 0,83^{\mathrm{a}}$ & $1,54 \pm 1,10^{\mathrm{b}}$ & $1,54 \pm 1,43^{\mathrm{b}}$ \\
\hline
\end{tabular}

Não foi observada diferença significativa para índice viscerossomático, índice hepatossomático entre os tratamentos, o que sugere que a manipulação do fotoperíodo, provavelmente, não influenciou no gasto energético destinado ao desenvolvimento gonadal (Tabela 2). No entanto, no estudo de Taranger et al. (2006) machos e fêmeas de bacalhau do Atlântico (Gadus morhua) sob regime de luz contínua (LL) apresentaram maiores valores de IHS em relação ao regime de luz natural (LN), indicando que o grupo de LL investiu menos energia para a reprodução. Ribeiro et al. (2006) também constataram em Steindachnerina (Characiforme: Curimatidae) diferenças no IHS, que diminuiu da fase de repouso para a fase de maturação.

Após 60 dias de experimento não foi observado diferença significativa para o peso da gônada. O IGS foi observado diferença significativa para o tratamento $24 \mathrm{~L}: 0 \mathrm{E}$ onde obteve menor valor em comparação aos demais tratamentos. Provavelmente, intensa atividade natatória durante todo o período de luz contínua levou a uma maior exaustão dos peixes, seguido de maior demanda energética e quebra de glicose em lactato pelo processo de glicólise anaeróbica no músculo branco a fim de atender as atividades motoras e não os processos reprodutivos. A maioria dos estudos envolvendo fotoperíodo e reprodução de peixes aponta uma relação entre o fotoperíodo e o eixo hipotálamo-hipófise-gonadal, refletido por alterações na espermatogênese e modificações nos níveis de hormônios hipofisários e esteróides ligados à reprodução.

Não foi observada diferente significativa para matéria seca,proteína bruta e extrato etéreo(Tabela 3). Apesar da não significativoo tratamento com $24 \mathrm{~L}: 0 \mathrm{E}$ obteve maior porcentagem de proteína com menor porcentagem de extrato etéreo.

Tabela 3: Média terro padrão da Matéria Seca (MS), Proteína bruta (PB) e Extrato etéreo (EE), de tilápia em função do fotoperíodo.

\begin{tabular}{cccc}
\hline Tratamento & 24L:0E & 12L:12E & 0L:24E \\
\hline MS (\%) & $23,38 \pm 1,06^{\mathrm{a}}$ & $24,1 \pm 1,55^{\mathrm{a}}$ & $23,5 \pm 1,45^{\mathrm{a}}$ \\
PB (\%) & $58,88 \pm 1,00^{\mathrm{a}}$ & $48,48 \pm 1,34^{\mathrm{a}}$ & $58,33 \pm 1,89^{\mathrm{a}}$ \\
EE (\%) & $17,84 \pm 1,44^{\mathrm{a}}$ & - & $21,57 \pm 1,977^{\mathrm{a}}$ \\
\hline
\end{tabular}

*Médias seguidas de letras diferentes diferem a $5 \%$ de probabilidade pelo teste de Duncan. 


\section{Conclusão}

Conclui-se que o fotoperíodo (12L:12E) promove melhor índice gonadossomático em tilápia. Para melhor qualidade de carcaça e desempenho produtivo sugere-se o fotoperíodo (24L:0E). Essas informações poderão nortear novos experimentos, melhorar as condições de cultivo, valorizar a atividade no sentido econômico, assim como, aumentar o número de alevinos destinados à aquicultura.

\section{Referências}

ADAMANTE, W. B. Estresse de alevinos de Dourado e Mandi sob diferentes densidades e tempos de transporte. 2005. 39 p. Dissertação (Mestrado). Universidade Federal de Santa Catarina, Florianópolis - SC. 2005.

AGOSTINHO, A. A.; NARAHARA M. Y.; GODINHO, H. P. Morfologia dos ovários de Plecostomus commersonii (Valenciennes, 1840) Osteichthyes-Loricariidae: desenvolvimento dos ovócitos e escala de maturidade.

Revista Brasileira de Biologia, v. 42, n. 1, p. 71-77, 1982.

AGOSTINHO, C. A. et al. Ciclo reprodutivo e primeira maturação sexual de fêmeas do lambari, Astyanax bimaculatus (L.) (Osteichthyes - Characidae) do rio Ivaí, Estado do Paraná. Revista Brasileira de Biologia, v. 44, n. 1, p. 31-36, 1984

AMANO, M. et al. Roles of melatonin in gonadal maturation of under yearling precocious male Masu Salmon. General and Comparative Endocrinology, v. 120, n. 2, p. 190-197, 2000.

AMANO, M et al. Effects of photoperiod on gonadotropinreleasing hormone levels in the brain and pituitary of underyearling male barfin flounder. Fisheries Science, v. 70, n. 5 , p. $812-818,2004$

\section{BALDISSEROTTO, B. Fisiologia de peixes aplicada à piscicultura.Santa Maria: UFSM, 2002, 212p.}

BANI A. et al. Effects of different photoperiods on growth, stress and haematological parameters in juvenile great sturgeon Huso huso. Aquaculture Research, v. 40, n. 16, p. 1899-1907, 2009.

BISWAS, A. et al. Growth and stress response in adult red sea bream, Pagrus major (Temminck \& Schlegel) exposed to different photoperiods at spawning season. Aquaculture Research, v. 41, n. 3, p. 519-527, 2010.

DAVIE, A et al. Inhibition of sexual maturation in tank reared haddock (Melanogrammus aeglefinus) through the use of constant light photoperiods. Aquaculture, v. 270, p. 379-389, 2007.

FALCON, J. et al. Current knowledge on the melatonin system in teleost fish. General and Comparative Endocrinology, v. 165, p. 469-482, 2010.
GINÉS, R. et al. The effects of long-day photoperiod on growth, body composition and skin colour in immature gilthead sea bream (Sparus aurata L.). Aquaculture Research, v. 35, n. 13, p. 1207-1212. 2004.

HANSEN, T. et al. Growth, gonadal development and spawning time of Atlantic cod (Gadus morhua) reared under different photoperiods. Aquaculture, v. 203, n. 1/3, p. 51-67, 2001

NAVARRO, F. K. S. P.; NAVARRO, R. D. Importância do fotoperíodo no crescimento e na reprodução de peixes. Revista Brasileira Reprodução Animal, v. 36, n. 1, p. 9499, 2012.

NAVARRO, F. K. S. et al. The effect of different photoperiods on plasma levels of $\mathrm{LH}$ and gonadal maturation of female lambari (Astyanax bimaculatus). Pesquisa Agropecuária Brasileira, v. 48, n. 8, p. 10641070, 2013.

NAVARRO, F. K. S. et al. Effect of photoperiod stress assessment and locomotor activity of female lambari (Astyanax bimaculatu). Ciência e Agrotecnologia, v. 38, n. 2, p. 173-180, 2014.

NAVARRO, R. D. et al. Effects of photoperiod on somatic growth and gonadal development in male nile tilapia. Acta Scientiarum: Technology (Online), v. 37, p. 381-385, 2015.

OPPEDAL, F. et al. Light intensity affects growth and sexual maturation of Atlantic salmon (Salmo salar) posts molts in sea cages. Aquatic Living Resources, v. 10, n. 7 , p. 351-357, 1997.

RAD, F.; BOZAOĐLU, S.; GÖZÜKARA, S. E. Effects of different long-day photoperiod son somatic growth and gonadal development in Nile tilapia (Oreochromis niloticus L.). Aquaculture, v. 255, p. 292-300, 2006.

RIBEIRO,V. M. A. et al. Ultrastructural changes in female hepatocytes during ovarian maturation of Steindachnerina (Pisces: Curimatidae). Brazilian Journal of Biology, v. 66, n. 4, p. 957-962, 2006.

TARANGER, G. L. et al. Continuous light delays sexual maturation and increases growth of Atlantic cod (Gadus morhua L.) in sea cages. Journal of Marine Science, v. 63, p. $365-375,2006$.
Recebido em: 04.02.2016 Aceito em: 01.08.2016 